\title{
Multi-Scale Simulations of Semiconductor Nanostructures
}

\author{
M. ZIELIŃSKI* \\ Instytut Fizyki UMK, Grudziądzka 5, 87-100 Toruń, Poland
}

\begin{abstract}
We demonstrate a multi-domain scheme for calculation of electronic and optical properties of semiconductor nanostructures. Three progressively smaller computational domains are used for strain simulation, single particle states calculation and computation of the Coulomb scattering matrix elements. Proposed approach offers a significant reduction of computational time and memory savings without sacrificing the accuracy of obtained spectra. We illustrate this method on the example of InAs/InP self-assembled quantum dots.
\end{abstract}

PACS: 73.21.La, 78.67.Hc, 71.15.-m, 71.15.Qe

\section{Introduction}

Atomistic modeling of optical and electronic properties of semiconductor nanostructures is a very demanding computational task. Up-to-date computers, even massively parallel machines, do not have satisfactory computational power allowing for an $a b$ initio treatment of million atom nanostructures such as quantum dots or quantum wires [1, 2]. For a practical solution the calculation is typically divided into set a smaller, subsequent calculations [3-10]: starting with strain field calculation (geometry optimization), followed by calculation of single particle levels and then calculation of the Coulomb scattering matrix elements as a necessary prerequisite for a final calculation of many-body excitonic spectra.

Strain field calculation is based on either continuum elasticity or atomistic approach [11], typically some flavor of valence force field method (VFF [12]). Single particle states are usually obtained with the effective mass [1], $k \cdot p$ method $[2,3]$ or atomistic semi-empirical approaches like empirical tight-binding (ETB [10, 13-15]) or empirical pseudopotentials method (EPM [4-6]). Finally, the Coulomb and optical matrix elements are calculated and excitation spectra for several quasi-particles (exciton, biexciton, trions) are calculated with the use of configuration interaction method $[1,7,10]$.

While continuous matter approaches undoubtedly demonstrated their usability in describing main spectral features of semiconductor quantum dots [1, 2], in recent years atomistic approaches have proven to be necessary for accurate description of the details of electronic and excitonic spectra of semiconductor nanosystems [16-18]. Contrary to continuous matter approaches, in the atomistic calculation the characteristic spatial resolution is not arbitrary, but is given by well defined atomic grid that cannot be reduced without altering system properties [11]

* e-mail: mzielin@fizyka.umk.pl (e.g. artificially reducing system symmetry). With domain size necessary for proper simulation of strain effects reaching 100 nanometers [19], computational domains for the strain simulation are on the order of $10^{8}$ atoms, presenting a significant challenge already at the level of the strain simulation. The use of modern multi-processor computers and parallelization of force field equations enables for attacking systems even up to several hundred million atoms, well beyond the size needed for the convergence of the strain simulation. However using large $\left(10^{8}\right)$ computational domains for the atomistic single particle levels calculation would be prohibitive, as for example the size of the tight-binding (TB) matrix would reach $\approx 10^{10} \times 10^{10}$ which is too large for a practical calculation. Fortunately, confined quantum dot states are by their nature predominately localized in the quantum dot area with relatively small (5-10\%) contribution in the surrounding matrix material. This allows for choosing smaller computational domain for the single particle calculation with number of atoms typically reaching up to $10^{6}$ atoms, resulting in significant memory saving and speed-up of entire calculation [19].

Once single particle states are calculated, the next step is the calculation of many-body spectra. This step can be further decomposed into two parts: calculation of the Coulomb (and optical) matrix elements and then the actual many-body calculation which is typically achieved by configuration interaction (CI) approach. With the proper parallelization scheme and under nearest (or second-nearest) neighbors approximation both the VFF and TB parts can scale nearly linearly with the number of atoms. Contrary, the calculation of the Coulomb matrix elements, even neglecting the contributions from three- and four-center integral, reduces to double summations $[8,10,15]$ over the (large) list of atoms in computational domain resulting in $\mathrm{O}\left(n^{2}\right)$ scaling. As for the sake of the following CI calculation one needs to calculate on the order of $10^{4}$ different Coulomb integrals this calculation turns to be the most time con- 
suming in the entire computation pipeline. In this paper we show that the size of the computational domain needed for the calculation of many body properties can be further reduced when compared to TB domain giving substantial savings in time of calculation and memory resources.

In the end we propose a scheme in which one uses three progressively smaller computational domains: the largest for the strain simulation, the intermediate for the single particle spectra, and finally the smallest for the calculation of the Coulomb matrix elements.

\section{Strain domain}

We illustrate our approach on the example of InAs / InP self-assembled disc-shaped quantum dot. The quantum dot height is $2.4 \mathrm{~nm}$ and its diameter is $18 \mathrm{~nm}$. The dot is situated on a $0.6 \mathrm{~nm}$ thick wetting layer. The computational domains at all scales were chosen to have cylindrical symmetry in order to match that of the quantum dot. The domain size is defined by its height (dimension parallel to the quantum dot growth direction) and radius.

Strain calculations were performed using VFF method described in detail in our earlier works [9, 10]. Atomic positions obtained in VFF step are afterwards used for the TB calculation $\left(s p^{3} d^{5} s^{*}\right.$ parameterization $\left.[14,20]\right)$.

Figure 1a shows the trace of strain tensor profiles in the dot area for several different VFF domain heights (radius fixed at $50 \mathrm{~nm}$ ). The larger the buffer is the more strain accumulates in the dot area and less in the surrounding buffer in agreement with analogous calculation by Lee et al. [19]. Once the buffer thickness is larger than $30 \mathrm{~nm}$, there is no noticeable difference in strain distribution. One can however argue whether the strain distribution is a good figure of merit and rather use single particle spectra calculated for different strain domain sizes as a reliable measure of convergence. Figure $1 b$ shows evolution of the single particle gap as a function of VFF domain thickness in vertical (growth) direction and domain thickness in quantum dot plane. Due to biaxial character of strain in flat, disc-like quantum dot and dominant confinement in growth ("vertical") direction, the system properties alter more with the change of the domain height rather than its radius. Despite the small height of quantum dot $(2.4 \mathrm{~nm})$ as compared to the domain height, choosing too small computational domain will result in existence of "unrelaxed" strain in the system causing shift of both electrons and holes on almost equal footing as shown in Fig. 1b. This is a manifestation of a long range character of strain effects and hence rather slow convergence with the change of the domain size.

Only the domain with the buffer thickness in radial direction on the order of $30 \mathrm{~nm}$ and buffer thickness in vertical thickness larger than $40 \mathrm{~nm}$ will guarantee that changes of single particle energies will be lower than $1 \mathrm{meV}$. Such a large domain size is in agreement with the work by Lee et al. [19], which might be surprising as we consider less (3\%) strained In As/InP system as compared

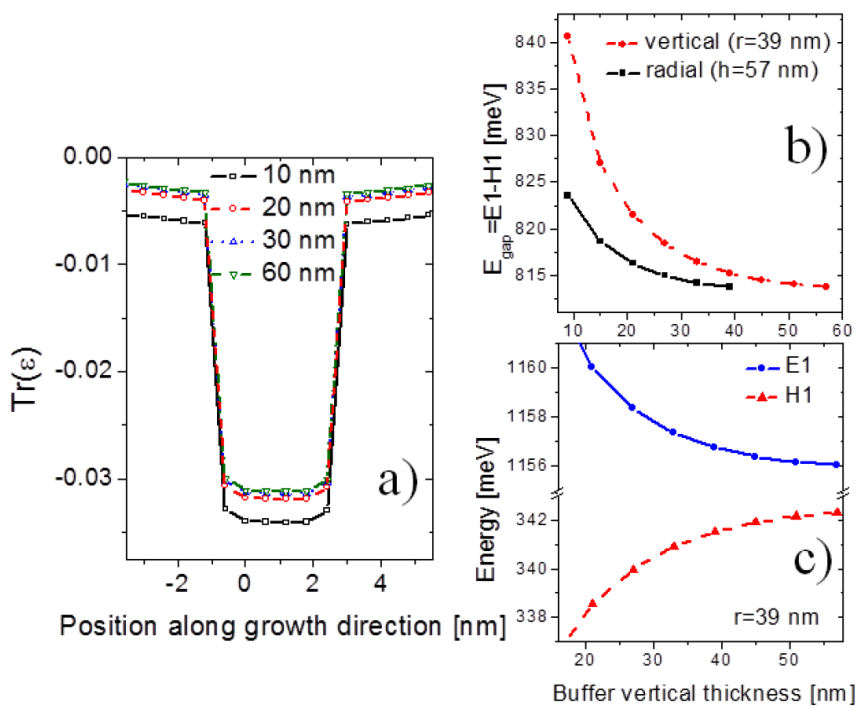

Fig. 1. Profile of strain tensor through the quantum dot center for different thicknesses of the VFF buffer in vertical direction (a). Evolution of single particle gap (b) as a function of buffer radial and vertical thickness. Evolution of electron $(E 1)$ and hole $(H 1)$ ground state energies (c) as a function of VFF domain vertical thickness.

to highly $(7 \%)$ strained InAs/GaAs systems considered in the above paper.

\section{Tight-binding domain}

Having established the converged VFF domain size, one can determine TB domain size again by varying domain radius or height. Figure 2 a shows evolution of single particle gap as function of the TB domain thickness in radial and vertical direction. Similarly to the VFF case, due to large confinement in the growth direction, the system properties are more altered by changing TB domain height (size in the growth direction), rather than the radius. However there is a noticeable difference between electron and hole states (Fig. 2b): due to the stronger confinement the holes single particle energies will converge faster. The domain thickness of about $5 \mathrm{~nm}$ in radial and vertical direction guarantees convergence of hole and electron ground states single particle energies below $0.1 \mathrm{meV}$. As the convergence rate with the TB domain size is related to the degree of the quantum state localization, the higher lying electron and holes states will demand larger domains and we note that should be taken into account when studying multi-excitonic effects involving highly excited single particle states.

\section{Coulomb matrix element domain}

The calculation of the Coulomb matrix elements (CME) consists of two contributions [8, 10]: the calculation of on-site terms which scales linearly with the 

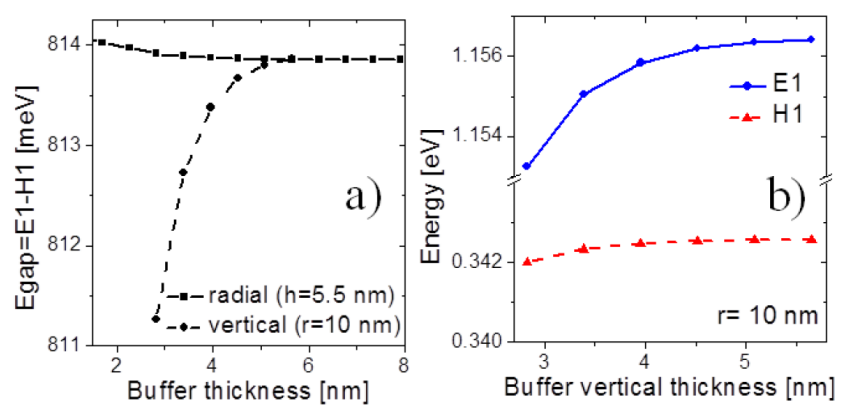

Fig. 2. Evolution of single particle gap $(E 1-H 1)$ as a function of TB domain thickness in radial and vertical direction (a). Evolution of electron ground $(E 1)$ and hole ground $(H 1)$ states energies as a function of TB buffer vertical thickness (b).

number of atoms and, the dominant, off-site contribution that scales like the square of the numbers of atoms in the domain. As for the study of multi-excitonic effects one needs to take into account many single particle states [7]; this results in a necessity of calculation of large number $\left(>10^{4}\right)$ of integrals presenting a formidable numerical task [7]. In order to reduce the numerical effort one can consider using smaller domain for CME calculation as compared to the TB case and verify validity of such approach by convergence tests.
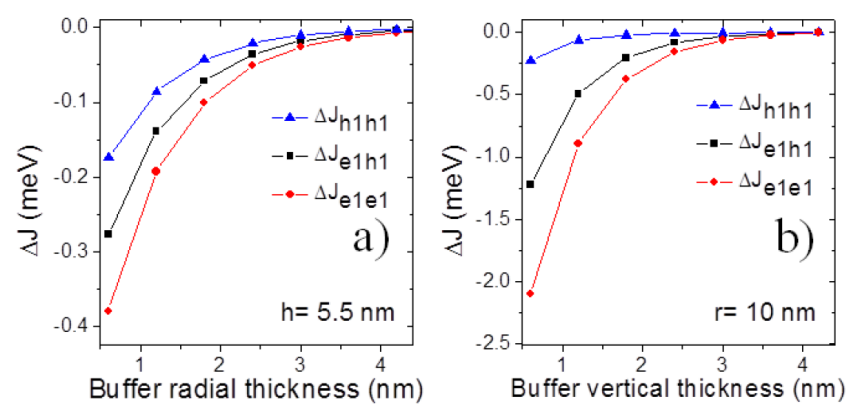

Fig. 3. Evolution of the electron-hole Coulomb energy $J_{e_{1} h_{1}}$ of the ground electron-hole $s$ state, electronelectron $J_{e_{1} e_{1}}$ and hole-hole $J_{h_{1} h_{1}}$ Coulomb energies as a function of domain thickness in radial (a) and vertical (b) direction.

Figure 3 shows the evolution of the electron-hole Coulomb energy $J_{e_{1} h_{1}}$ of the ground electron-hole $s$ state, electron-electron $J_{e_{1} e_{1}}$ and hole-hole $J_{h_{1} h_{1}}$ Coulomb energies as a function of domain thickness in vertical (a) and radial (b) direction. The convergence rate depends strongly on the level of localization of states contributing the integrals, thus integrals involving well localized holes will converge faster than those with electrons. Again there is a very noticeable difference between domain size in growth and lateral thickness. Buffer thickness of $2 \mathrm{~nm}$ in radial direction and $3 \mathrm{~nm}$ is already sufficient to guarantee convergence below $100 \mu \mathrm{eV}(\approx 0.5 \%$ accuracy). Such dimensions result in overall CME buffer size roughly half the size of the TB domain, resulting in four times faster calculation compared to full size CME due to the $\mathrm{O}\left(n^{2}\right)$ scaling. For the $1 \mathrm{meV}$ accuracy (5\%) in calculation of the Coulomb integrals savings could be even more substantial offering about 10 times speed-up.

\section{Conclusions}

We have demonstrated the multi-scale (multi-domain) approach for calculation of spectral properties of semiconductor nanosystems illustrated with the example of InAs/InP disk-like quantum dots. We have also verified our conclusion performing calculation for larger $(h=$ $3.0 \mathrm{~nm}$ ) elongated quantum dot (not shown here) and obtaining similar conclusions. We have shown that progressively smaller computation domains can be used for strain, single particle and Coulomb matrix elements calculation, with corresponding number of atoms: $20 \mathrm{mln}$, $0.33 \mathrm{mln}, 0.177 \mathrm{mln}$ guaranteeing convergence within assumed thresholds. For the considered system (flat, disc-shape quantum dot) we also found that it is more beneficial (in terms of introduced error) to reduce domain sizes in radial (lateral) direction, rather in vertical (growth) direction due to dominant system confinement in the latter. As the number of atoms grows proportionally to the square of the domain radius, such approach additionally results in having a smaller number of atoms in the domain. We have found that the apparent convergence in strain tensor distribution does not guarantee convergence in the corresponding single particle spectra. We notice that the Coulomb integrals calculated for better confined (hole) states converge faster with increasing domain size, than those of less confined (electron) states. To summarize, we propose a multi-scale approach that significantly reduces computational effort in calculation of single particle and many-body properties of semiconductor quantum dots.

\section{Acknowledgments}

This work was supported by Iuventus Plus program of the Polish Ministry of Science and Higher Education and by the Foundation for Polish Science, Homing Plus Programme co-financed by the European Union within the European Regional Development Fund.

\section{References}

[1] L. Jacak, P. Hawrylak, A. Wojs, Quantum Dots, Springer, Berlin 1998.

[2] D. Bimberg, M. Grundmann, N.N. Ledentsov, Quantum Dot Heterostructures, Wiley, New York 1998.

[3] O. Stier, M. Grundmann, D. Bimberg, Phys. Rev. B 59, 5688 (1999).

[4] A. Canning, L.W. Wang, A. Williamson, A. Zunger, J. Comp. Phys. 160, 29 (2000).

[5] A.J. Williamson, L.W. Wang, A. Zunger, Phys. Rev. B 62, 12963 (2000). 
[6] L. He, A. Zunger, Phys. Rev. B 73, 115324 (2006).

[7] W. Sheng, S.-J. Cheng, P. Hawrylak, Phys. Rev. B 71, 035316 (2005).

[8] S. Schulz, S. Schumacher, G. Czycholl, Phys. Rev. B 73, 245327 (2006).

[9] W. Jaskólski, M. Zieliński, G.W. Bryant, J. Aizpurua, Phys. Rev. B 74, 195339 (2006).

[10] M. Zieliński, M. Korkusiński, P. Hawrylak, Phys. Rev. B 81, 085301 (2010).

[11] C. Pryor, J. Kim, L.W. Wang, A.J. Williamson, A. Zunger, J. Appl. Phys. 83, 2548 (1998).

[12] P.N. Keating, Phys. Rev. 145, 637 (1966); R.M. Martin, Phys. Rev. B 1, 4005 (1970).

[13] K. Leung, K.B. Whaley, Phys. Rev. B 56, 7455 (1997).

[14] J.M. Jancu, R. Scholz, F. Beltram, F. Bassani, Phys. Rev. B 57, 6493 (1998).
[15] S. Lee, L. Jönsson, J.W. Wilkins, W. Bryant, G. Klimeck, Phys. Rev. B 63, 195318 (2001).

[16] R. Singh, G. Bester, Phys. Rev. Lett. 103, 063601 (2009).

[17] M. Korkusiński, M. Zieliński, P. Hawrylak, J. Appl. Phys. 105, 122406 (2009).

[18] G. Bester, A. Zunger, Phys. Rev. B 71, 045318 (2005).

[19] S. Lee, F. Oyafuso, P. von Allmen, G. Klimeck, Phys. Rev. B 69, 045316 (2004)

[20] M. Zieliński, Coupling strain into atomistic, tight-binding Hamiltonian, submitted for publication in Phys. Rev. B. 